\title{
Will women's choice of position in first stage of labor affect labor process?
}

\author{
Shubha Rao*, Shylabhirami Sridharan, Akhila Vasudeva, Roopa P. S.
}

\author{
Department of Obstetrics and Gynecology, Kasturba Medical College, Manipal, Manipal Academy of Higher \\ education (MAHE), Karnataka, India
}

Received: 08 February 2020

Accepted: 02 March 2020

*Correspondence:

Dr. Shubha Rao,

E-mail: shubharao257@gmail.com

Copyright: (C) the author(s), publisher and licensee Medip Academy. This is an open-access article distributed under the terms of the Creative Commons Attribution Non-Commercial License, which permits unrestricted non-commercial use, distribution, and reproduction in any medium, provided the original work is properly cited.

\begin{abstract}
Background: Controlling the process of childbirth has disabled the parturient to embrace the most spontaneous position of delivery but constricting her to assume a recumbent position. Objective of this study was to study if alternating comfortable maternal positioning i.e., recumbent and alternative position have any influence in the process of labor, type of delivery, neonatal well-being.

Methods: Study conducted an observation study on term pregnant women. Study inclusion criteria included all term pregnant women. Exclusion criteria included multiple pregnancies, preterm patient, severe pre-eclampsia, and eclampsia, preterm premature rupture of membranes, sever intrauterine growth restricted fetus. The measured date were maternal general characteristics, duration of labor process, type of delivery and neonatal outcome. Patients were divided into two groups. Group A - if they spent more than $50 \%$ in a recumbent position and Group B - any other alternating position.

Results: A total 250 women were equally included in this study. The demographic characteristics were matched in both groups and found no significant difference. In the process of labor, Group B had a difference of 1 hours as compared to Group A and the rate of cervical dilation was also faster in Group B. Both of these variables were found to be statistically significant. However, there were no significant difference in the terms of type of delivery and neonatal outcome.

Conclusions: The ancient practice of recumbent position during labor is to be discarded as alternating maternal position during the process of labor may a positive influence on the total duration labor. However even though it may or may not have an influence on the other outcome such as route of delivery and neonatal outcome, it is best to encourage women to move and deliver in the most comfortable position.
\end{abstract}

Keywords: Labor process, Maternal position

\section{INTRODUCTION}

All through the years in human cultures women from all over the world have preferred to give birth with their bodies in sitting or squatting by grasping a rod or a companion, ropes etc. and usually do not prefer lying flat on their back positions. However in the present era, in developing countries, there has been an ongoing obstetrical practice that once a labouring woman has had a hospital admission it is imperative that she is stranded in the supine position and restrain from any spontaneous activity that involves her ambulating. ${ }^{1,2}$ This is in assumption that it would be easier to monitor the fetal wellbeing and process of labor followed by the administration of analgesia if required. ${ }^{2,3}$ In the present literature there has never been any consensus regarding the best position of labor and its agreement on the betterment of maternal-fetal outcome although the 
recumbent position if known to be convenient for the staff. $^{1,4,5}$ However this position may make the birth process complicated and strenuous.

The upright position makes us unique amongst mammals. However we do not even take the advantage of the help that gravity can provide as an upright position is associated with more effective uterine contractions, better alignment of birth canal, increase pelvic outlet diameters and thereby reducing maternal and neonatal morbidity. ${ }^{6-10}$ In contrast the recumbent position is best suited to palpate for uterine contraction and perform vaginal examination and also assess the fetal heart rate, but it is also known to cause aortocaval compression, less effective uterine contractions, longer duration of labor, more chances of operative deliveries and more request of analgesia. ${ }^{11-14}$ It is reasonable to believe that lack of birthing position as hospital may be one of the contributory factor to women choosing to give birth in recumbent positions.

The aim of this study was to categorize the patient as those who spend more than $50 \%$ of labor in recumbent position to those who spend more than $50 \%$ of labor in alternating position in terms of intra partum, maternofetal and neonatal outcome.

\section{METHODS}

This was an observational study in term pregnant woman admitted to the labor room in a tertiary care hospital in South India from August 2017 to July 2018. All the patients were informed about the study and consented for the same. According to the criteria of labor, presence of regular uterine contractions and second stage of labor was defined with full dilatation of cervix. ${ }^{15}$

\section{Inclusion criteria}

- Term pregnant woman at labor onset, a Transabdominal ultrasound was done to confirm the fetal position.

\section{Exclusion criteria}

- All cases of multiple pregnancies, preterm labor, severe pre-eclampsia, eclampsia and severe IUGR where the associated co morbidity may alter the mode of delivery chosen or restrict the ambulation.

Intra partum date was collected on a partogram. For all woman, general characteristics such as age, BMI, gestational age, labor process i.e., duration of first stage and second stage of labor, analgesia required and mode of delivery (spontaneous, operative vaginal delivery or cesarean section), neonatal outcome at birth Apgar score at birth and 5 minutes. Group A - included patients who spent more than $50 \%$ of their labor in lying down position and Group B included patient who spent more than 50\% of their labor in any other alternative position i.e., sitting or squatting. All patients were monitored according to protocol as described in intra partum care for healthy women and babies in NICE guidelines. ${ }^{16}$ Pain intensity was assessed by determining the analgesia requested by the patient.

The primary objective of this study is determining the outcome between two groups in terms of duration of labor and progress of labor. Secondary outcome is to compare the maternal analgesia request, mode of delivery i.e., operative vaginal delivery or by caesarean section.

\section{Statistical analysis}

Statistical analysis was performed using SPSS software. Statistical significance was defined as $\mathrm{p}<0.05$.

\section{RESULTS}

Study recruited a total of 250 women in this study. Group A - had 125 patients and Group B had 125 patients. The mean age in Group A was $30.52 \pm 2.55$ years and the mean age in Group B was 30.48 \pm 3.24 years. Mean BMI in Group A was 25.24 \pm 3.3 and in Group B was 25.52 \pm 3.6 . None of these findings were proven to be statistically significant. Group A and Group B were both matched for general maternal characteristics (Table 1).

In Group A, $85(68 \%)$ cases had vaginal delivery, of which $3(3.5 \%)$ cases had assistance with forceps and 3 $(3.5 \%)$ cases has assistance with vacuum. $40(32 \%)$ cases underwent caesarean section. In Group B, there were more number of vaginal deliveries i.e. $92(73.6 \%)$ of which $4(4.3 \%)$ cases had assistance with forceps and 4 $(4.3 \%)$ cases had assistance with vacuum.

Table 1: Demographic characteristics.

\begin{tabular}{|llll|}
\hline Demography & Group 1 $(\mathbf{n}=\mathbf{1 2 5})$ & Group 2 $(\mathbf{n}=\mathbf{1 2 5})$ & p value $(<\mathbf{0 . 0 5}$ is significant $)$ \\
\hline Maternal age $($ mean \pm SD) & $30.52 \pm 2.55$ & $30.48 \pm 3.24$ & 0.25 \\
\hline BMI (mean \pm SD) & $25.24 \pm 3.3$ & $25.51 \pm 3.6$ & 0.28 \\
\hline Parity & & & \multirow{2}{*}{0.70} \\
\hline Primigravida & 60 & 63 & 0.497 \\
\hline Multigravida & 65 & 62 & \\
\hline Gestational age (weeks) & $38.5 \pm 0.2$ & $38.2 \pm 0.4$ & \multirow{2}{*}{$0.4 \%)$} \\
\hline Occipital posterior position & $7(5.6 \%)$ & $4(3.4 \%)$ & \\
\hline
\end{tabular}


Group B also had less number of caesarean section i.e., $33(26.4 \%)$ cases. Even though there were more number of vaginal deliveries and lesser number of caesarean section, this difference did not prove to be statistically significant.

Statistically significant differences were found in duration of labor of both the first and second stage of labor (mean 5.22 \pm 1.98 hours in Group A and 4.26 \pm 2.18 hours in Group B), a difference of almost 1 hours. ( $p$ value $<0.05$ ). The mean duration of first stage of labor in Group A was $235 \pm 107$ minutes and $206.34 \pm 121$ minutes in Group B. The mean duration of second stage of labor in Group A was 53.7 \pm 22.2 minutes and $48.6 \pm 18.12$ minutes in group B. Study also found the rate of cervical dilation was more in Group A than in Group B (mean $1.50 \pm 0.64$ in Group A and $1.78 \pm 0.74$ in Group B) which was found to be statistically significant (Table 2). However, both groups had no significant different in the requirement of analgesia.

Considering only Group B patients, no significant difference was found in the duration of labor comparing alternative positions (Table 3 ) however more number of patients 58 who adopted the propped position had normal vaginal delivery which was found to be statistically significant (Table 4).

Table 2: Comparison between the groups in terms of duration of labor and need for analgesia.

\begin{tabular}{|ll|ll|}
\hline \multicolumn{1}{|c|}{ Outcomes } & Group 1 $(\mathbf{N}=\mathbf{1 2 5})$ & Group 2 $(\mathbf{N}=\mathbf{1 2 5})$ & p value $(<0.05$ is significant $)$ \\
\hline Duration of labor(hours) & $5.22 \pm 1.98$ & $4.26 \pm 2.18$ & 0.024 \\
\hline Stage 1 (min) mean \pm SD & $206.34 \pm 121$ & $235 \pm 107$ & 0.033 \\
\hline Stage 2 (min) mean \pm SD & $48.6 \pm 18.12$ & $53.7 \pm 22.2$ & 0.032 \\
\hline Rate of cervical dilatation (cm/hour) & $1.50 \pm 0.64$ & $1.78 \pm 0.74$ & 0.007 \\
\hline Requirement of analgesia & 18 & 10 & 0.10 \\
\hline
\end{tabular}

Table 3: Comparison in Group B patients and their total duration of labor.

\begin{tabular}{|l|llll|}
\hline Outcomes & Propped up & Walking & Sitting & p value $(<0.05$ is significant $)$ \\
\hline Stage 1 $(\mathrm{min})$ mean \pm SD & $190.85 \pm 114.32$ & $222.60 \pm 117.5$ & $217.85 \pm 139.39$ & 0.45 \\
\hline Stage 2 $(\mathrm{min})$ mean \pm SD & $48.08 \pm 19.78$ & $51.30 \pm 15.16$ & $45.07 \pm 17.09$ & 0.48 \\
\hline Rate of cervical dilatation (cm/hour) & $1.85 \pm 0.80$ & $1.59 \pm 0.65$ & $1.83 \pm 1.09$ & 0.44 \\
\hline
\end{tabular}

Table 4: Comparison of mode of delivery in Group B patients.

\begin{tabular}{|c|c|c|c|c|}
\hline Outcomes & Propped up & Walking & Sitting & $p$ value ( $<0.05$ is significant) \\
\hline Normal delivery & 58 & 10 & 16 & \multirow{4}{*}{0.04} \\
\hline Assisted delivery A. Forceps & 1 & 2 & 1 & \\
\hline B. Vaccum & 2 & 0 & 2 & \\
\hline Caesarean section & 14 & 9 & 10 & \\
\hline
\end{tabular}

There were no significant differences found in the neonatal outcome of both Group A and Group B as determined by Apgar score at birth and 5 minutes.

\section{DISCUSSION}

The journey of pregnancy is not a straight road as there are many bends and curves which can make it an unpleasant one. Of them, the most crucial part of pregnancy that many women fear about is that of childbirth. The reason behind this could be due to lack of knowledge about it. A pleasant experience during childbirth is subjective to many factors such as perception to pain, health care support, motivation, and support by relatives. One of the factors that may have a positive influence on the whole experience is a change in the position in labor. ${ }^{17}$ There have been many disadvantages of recumbent position, few being the uterine hypo perfusion associated with it, less effectiveness of uterine contractions and the fetal alignments to the pelvic dimensions. ${ }^{14}$ A recent meta-analysis has stated that vertical positions have lesser analgesia request, reduced labor length, necessity of interventions thereby making the labor process more comfortable for the women in labor. ${ }^{4,18,19}$ Interventions such as amniotomy, oxytocin augmentation or monitoring of fetal condition and uterine contractions are more difficult in vertical positions than in recumbent position which is why most facilities offer recumbent positions. ${ }^{19}$ Contrary to the above, several studies have reported that a laboring woman may have a shorter duration of labor, avoiding augmentation if a comfortable position is allowed with good motivation from the health support and from the family members. $8,9,13,20$ 
Till date, there have been no definitive evidence to define the real role of position in labor. ${ }^{1,4,5}$ In this study however we found that in the patients assuming alternative positions other than recumbent had shorter duration of labor probably confirming that effect of gravity in favoring uterine contractions and fetal alignment at birth canal. The vertical position has an advantage of gravity, and increase in the size of pelvic diameter thanks to the nutation movement and to the coccyx re-propulsion thereby making the labor process less painful and more effective uterine contractions. The sitting position has an advantage on gravity by increasing the pelvic diameter and fetal alignment to the pelvis but has a disadvantage by increasing the pressure on the sacrum with a risk for perineal trauma. The 'all fours position' reduces the effect of gravity and uterine contraction and is known to be the best position for correcting fetal malposition to reduce the cervical edema and sacral pressure of the presenting part, it thereby increases the anteroposterior diameter in the expulsive phase. The squatting position uses gravity, there is an increase on the pelvic diameters and expedites fetal descent. ${ }^{19,21}$ This study did not a significant difference in the duration of labor in patients who opted for position other than recumbent position this could probably due to limitation of sample size however study found a statistical significant difference in the mode of delivery in the patients who opted for position other than recumbent position, mainly patients who were in propped up position had more number of vaginal deliveries similar to study done by Gizzo et al assuming vertical position during delivery had a positive outcome in the mode of delivery and duration of labor. ${ }^{22}$ However this study also had limitation in the variety of position offered as Gizzo et al, included "all fours position" and squatting position and proved that adopting "all fours position" has an impact on the rotation of head. ${ }^{22}$

In spite of various studies due to absence of strong evidences, it is unreasonable to impose a laboring position different from the spontaneous one. ${ }^{23}$ Although Golara et al proved that maternal immobilization may be a causative factor for shoulder dystocia, in the presence of high risk pregnancy or continuous intra partum care the use of alternative positions should be carefully evaluated. ${ }^{24-28}$ However there should be further evidence to prove it, in the absence of any antepartum/intra partum complications with the mother or the fetus, it is imperative that all women should be encourage for alternative positions in labor for an easier and a comfortable labor process.

\section{CONCLUSION}

Pregnancy is the most natural process that a woman goes through in her life and ensuring that she has the most comfortable and pleasant time is the duty of her caretakers. A simple sitting position for the women in labor may go a long way in reducing the discomfort by shortening the duration of labor and also help in the mode of delivery and provide a safe birthing experience.
However, in situations when strict monitoring of fetal wellbeing is required or intensive intra partum care are necessary, the use of alternative positions should be carefully considered. But in the absence of any such indications, the most natural and spontaneous position should be adopted for the betterment of maternal wellbeing.

Funding: No funding sources Conflict of interest: None declared Ethical approval: Not required

\section{REFERENCES}

1. Gupta JK, Sood A, Hofmeyr GJ, Vogel JP. Position in the second stage of labour for women without epidural anaesthesia. Cochrane Database Syst Rev. 2017;5:CD002006.

2. Jonge A de, Rijnders MEB, Diem MT van, Scheepers PLH, Lagro-Janssen ALM. Are there inequalities in choice of birthing position?: Sociodemographic and labour factors associated with the supine position during the second stage of labour. Midwifery. 2009;25(4):439-48.

3. Thies-Lagergren L, Hildingsson I, Christensson K, Kvist LJ. Who decides the position for birth? A follow-up study of a randomised controlled trial. Women Birth. 2013;26(4):e99-104.

4. Lawrence A, Lewis L, Hofmeyr GJ, Dowswell T, Styles C. Maternal positions and mobility during first stage labour. Cochrane Database Syst Rev. 2009;15(2):CD003934.

5. Kemp E, Kingswood CJ, Kibuka M, Thornton JG. Position in the second stage of labour for women with epidural anaesthesia. Cochrane Database Syst Rev. 2013;1:CD008070.

6. Thilagavathy G. Maternal birthing position and outcome of labor. J Family Welfare. 2012;58:6.

7. Souza JP, Miquelutti MA, Cecatti JG, Makuch MY. Maternal position during the first stage of labor: a systematic review. Reprod Health. 2006;3(1):10.

8. Chang SC, Chou MM, Lin KC, Lin LC, Lin YL, Kuo SC. Effects of a pushing intervention on pain, fatigue and birthing experiences among Taiwanese women during the second stage of labour. Midwifery. 2011;27(6):825-31.

9. Ben Regaya L, Fatnassi R, Khlifi A, Fékih M, Kebaili S, Soltan K, et al. Intérêt de la déambulation au cours du travail obstétrical: étude prospective randomisée de 200 cas. J Gynécol Obstét Biol Reprod. 2010;39(8):656-62.

10. Walker C, Rodríguez T, Herranz A, Espinosa JA, Sánchez E, Espuña-Pons M. Alternative model of birth to reduce the risk of assisted vaginal delivery and perineal trauma. Int Urogynecol J. 2012;23(9):1249-56.

11. Jonge AD, Lagro-Janssen ALM. Birthing positions. A qualitative study into the views of women about various birthing positions. J Psychosomat Obstet Gynecol. 2004;25(1):47-55. 
12. Jonge AD, Teunissen TAM, Lagro-Janssen ALM. Supine position compared to other positions during the second stage of labor: a meta-analytic review. J Psychos Obstet Gynecol. 2004;25(1):35-45.

13. Nilsen E, Sabatino H, Lopes MHB de M. Dor e comportamento de mulheres durante o trabalho de parto e parto em diferentes posições. Revista da Escola de Enfermagem da USP. 2011;45(3):557-65.

14. Gupta JK, Nikodem C. Maternal posture in labour. Eur J Obstet Gynecol Reprod Biol. 2000;92(2):2737.

15. Cunningham FG. Normal labor and delivery. Williams Obstet. 1997;261-317.

16. Nunes VD, Gholitabar M, Sims JM, Bewley S. Intrapartum care of healthy women and their babies: summary of updated NICE guidance. BMJ. 2014;349:g6886.

17. Nieuwenhuijze MJ, Jonge A de, Korstjens I, Budé L, Lagro-Janssen TLM. Influence on birthing positions affects women's sense of control in second stage of labour. Midwifery. 2013;29(11):e107-14.

18. Hunter S, Hofmeyr GJ, Kulier R. Hands and knees posture in late pregnancy or labour for fetal malposition (lateral or posterior). Cochrane Database Syst Rev. 2007;4:CD001063.

19. Zwelling E. Overcoming the challenges: maternal movement and positioning to facilitate labor progress. MCN Am J Matern Child Nurs. 2010;35(2):72-8.

20. Thies-Lagergren L, Kvist LJ, Christensson K, Hildingsson I. No reduction in instrumental vaginal births and no increased risk for adverse perineal outcome in nulliparous women giving birth on a birth seat: results of a Swedish randomized controlled trial. BMC Preg Childbirth. 2011;11(1):22.

21. Hanson L. Second-stage labor care: challenges in spontaneous bearing down. The J Perinat Neonat Nurs. 2009;23(1):31.

22. Gizzo S, Di Gangi S, Noventa M, Bacile V, Zambon A, Nardelli GB. Women's choice of positions during labour: return to the past or a modern way to give birth? a cohort study in Italy. Biomed Res Inter. 2014:2014.

23. Desbriere R, Blanc J, Le Dû R, Renner J-P, Carcopino X, Loundou A, et al. Is maternal posturing during labor efficient in preventing persistent occiput posterior position? A randomized controlled trial. Am J Obstet Gynecol. 2013;208(1):60.e1-8.

24. Golara M, Plaat F, Shennan AH. Upright versus recumbent position in the second stage of labour in women with combined spinal-epidural analgesia. Inter J Obstet Anesth. 2002;11(1):19-22.

25. Berretta R, Gizzo S, Dall'Asta A, Mazzone E, Monica M, Franchi L, et al. Risk of preterm delivery associated with prior treatment of cervical precancerous lesion according to the depth of the cone. Disease Markers. 2013;35(6):721-6.

26. Gizzo S, Patrelli TS, Rossanese M, Noventa M, Berretta R, Di Gangi S, et al. An Update on Diabetic Women Obstetrical Outcomes Linked to Preconception and Pregnancy Glycemic Profile: A Systematic Literature Review. The Scient World J. 2013:2013.

27. Patrelli TS, Gizzo S, Cosmi E, Carpano MG, Gangi SD, Pedrazzi G, et al. Maternal Hydration Therapy Improves the Quantity of Amniotic Fluid and the Pregnancy Outcome in Third-Trimester Isolated Oligohydramnios. J Ultrasound Med. 2012;31(2):239-44.

28. Zanardo V, Fanelli T, Weiner G, Fanos V, Zaninotto $\mathrm{M}$, Visentin $\mathrm{S}$, et al. Intrauterine growth restriction is associated with persistent aortic wall thickening and glomerular proteinuria during infancy. Kidney Inter. 2011;80(1):119-23.

Cite this article as: Rao S, Sridharan S, Vasudeva A, Roopa PS. Will women's choice of position in first stage of labor affect labor process?. Int J Reprod Contracept Obstet Gynecol 2020;9:1515-9. 